\title{
Infrapatellar Fat Pad-Derived Stem Cell-Based Regenerative Strategies in Orthopedic Surgery
}

\author{
Pinar Yilgor Huri, $\mathrm{PhD}^{1}$, Seren Hamsici, $\mathrm{MSc}^{1}$, Emre Ergene, $\mathrm{MSc}^{1,2}$, Gazi Huri, $\mathrm{MD}^{3}$, and \\ Mahmut Nedim Doral, $\mathrm{MD}^{3}$ \\ ${ }^{1}$ Department of Biomedical Engineering, Ankara University Faculty of Engineering, Ankara; ${ }^{2}$ Ankara University Biotechnology Institute, Ankara; ${ }^{3}$ Department of \\ Orthopedics and Traumatology, Hacettepe University Faculty of Medicine, Ankara, Turkey
}

\begin{abstract}
Infrapatellar fat pad is a densely vascularized and innervated extrasynovial tissue that fills the anterior knee compartment. It plays a role in knee biomechanics as well as constitutes a source of stem cells for regeneration after knee injury. Infrapatellar fat pad-derived stem cells (IPFP-ASCs) possess enhanced and age-independent differentiation capacity as compared to other stem cells, which makes them a very promising candidate in stem cell-based regenerative therapy. The aims of this review are to outline the latest advances and potential trends in using IPFP-ASCs and to emphasize the advantages over other sources of stem cells for applications in orthopedic surgery.
\end{abstract}

Keywords: Knee, Fat pad, Stem cell

\section{Stem Cell-Based Therapy}

The novel field of regenerative medicine has recently expanded with the use of stem cells as effective therapeutic tools for orthopedic surgery ${ }^{1,2)}$. Inherent self-renewal properties of stem cells and their differentiation potential into the multiple lineages hold promise to overcome the limitations of current therapeutic options. An ideal stem cell source should be easily accessible by a minimally invasive procedure; the procedure should be cost-effective for clinical translation, and the isolated cells should safely encompass autologous and/or allogeneic therapies; moreover, the

Received August 4, 2017; Revised (1st) November 22, 2017;

(2nd) December 22, 2017; Accepted January 5, 2018

Correspondence to: Gazi Huri, MD

Department of Orthopedics and Traumatology, Hacettepe University

Faculty of Medicine, Ankara, Turkey

Tel: +90-312-305-1209, Fax: +90-312-305-5000

E-mail: gazihuri@hacettepe.edu.tr

Source of funding: This study was supported by Scientific and

Technological Research Council of Turkey (214M160) and Turkish

Academy of Sciences for support of PYH (TUBA-GEBIP Program).

This is an Open Access article distributed under the terms of the Creative Commons Attribution Non-Commercial License (http://creativecommons.org/licenses/by-nc/4.0/) which permits unrestricted non-commercial use, distribution, and reproduction in any medium, provided the original work is properly cited. stem cells should have high proliferative capacity with a high potential for differentiation into multiple lineages in a controllable and reproducible manner.

\section{Adipose Tissue as a Source of Stem Cells}

Mesenchymal stem cells (MSCs) were originally identified within bone marrow and their multipotency generates a great deal of interest ${ }^{3)}$. However, the low amount of MSCs harvested from the bone marrow (bone marrow-derived mesenchymal stem cells [BMSCs]) through an invasive aspiration procedure created the need for further ex vivo expansion. In this sense, multipotent stem cells derived from adipose tissue with similar characteristics to BMSCs, called adipose-derived stem cells (ASCs), were found to be a more feasible and promising candidate for stem cell pool ${ }^{4)}$. This promise emanated from such properties of ASCs including their abundance in human body, their ease of accessibility and simpler isolation procedure as compared to aspiration from bone marrow, resulting in less patient discomfort and less risk associated with the surgical procedure ${ }^{5,6)}$. Moreover, ASCs have the capacity to proliferate to a higher degree as compared to BMSCs with a greater rate of stem cell marker expression ${ }^{7,8)}$. Furthermore, it has been shown that ASCs are more resistant to apoptosis than BMSCs in in vitro conditions ${ }^{9}$. ASCs have also shown to possess 
higher oncogenic resistance (leading to less risk of malignant transformation) as compared to BMSCs ${ }^{10)}$, which makes them promising candidates for cell transplantation therapies.

Along with these, many studies have indicated the immunosuppressive effects of MSCs derived from different sources, including $\mathrm{ASCs}^{11,12)}$. For example, the immunomodulatory capacities of BMSCs and ASCs obtained by one step cell concentration (stromal vascular fraction [SVF]) from age-matched donors were compared and it was shown that cells from SVF have more potent immunomodulatory effects which is related with higher levels of cytokine secretion ${ }^{13)}$. In another study, ASCs were shown to display a higher rate of $\mathrm{B}$ cell inhibition compared to BMSCs ${ }^{11}$. Even though MSCs derived from either source were capable of inhibiting NK and T cell activation, ASCs indicated a higher overall inhibitory effect ${ }^{14}$. Overall, ASCs are characterized by immunosuppressive properties and low immunogenicity ${ }^{6}$.

\section{Infrapatellar Fat Pad-Derived Stem Cells}

ASCs used in cell therapy are obtained mostly from subcutaneous adipose tissue by liposuction (SC-ASCs). On the other hand, the potential of ASCs isolated from infrapatellar fat pad (IPFP-ASCs), which is located in close proximity to the synovial membrane, in knee joint cartilage repair has fueled great interest in their therapeutic potential. The main promise of IPFP-ASCs over other MSC sources is that these cells show age-independent proliferation and differentiation potential, whereas others generally possess age-related decrease in these properties ${ }^{15}$. Moreover, IPFP-ASC's significant chondrogenic potential makes them promising candidates for exploration of use in other tissue regenerative approaches ${ }^{16)}$.

\section{Isolation and Characterization}

IPFP is routinely removed as surgical waste during knee arthroplasty, and it represents an accessible source of ASCs. In 1904, Hoffa described the surgical removal of IPFP, and after that Kumar mentioned an arthroscopic technique to ablate IPFP in $2007^{17}$. In brief, for the isolation of the IPFP during knee arthroplasty in our clinical setting, the tourniquet was inflated and then limited medial parapatellar skin incision (ca. $10 \mathrm{~cm}$ in length) was performed beginning $2 \mathrm{~cm}$ proximal to the superior pole of the patella and passing along the medial border of the patella to the medial border of the tibial tubercle. Afterwards, the IPFP was exposed and excised. This excised IPFP can be directly minced and the emerging stromal fraction including the stem cells can be directly used in the regeneration of bone, cartilage or soft tissues within the knee during surgery. For the isolation of ASCs from the excised IPFP, the tissue was transferred to the laboratory and the following procedure was applied under sterile conditions. IPFP was washed with sterile phosphate-buffered saline (PBS) to remove the blood and then was mechanically chopped into small pieces. The tissue was then digested enzymatically $(0.1 \%$ collagenase type I, $1 \%$ bovine serum albumin and $2 \mathrm{mM} \mathrm{CaCl}_{2}$ within sterile PBS) for 1 hour at $37^{\circ} \mathrm{C}$ under constant agitation within $50 \mathrm{~mL}$ tubes. Tubes were then centrifuged and the pellet was resuspended in PBS. Cell suspension was passed through a cell strainer and cells were collected in fresh tubes. After a second centrifugation step to remove the remaining fatty constituents, cell pellet was suspended in cell culture media including high glucose Dulbecco's Modified Eagle Medium (DMEM) supplemented with $10 \%$ fetal bovine serum, $1 \%$ antibiotics and $1 \mathrm{ng} / \mathrm{mL}$ FGF-2, cultured normally and stored within liquid nitrogen.

The independence of age-related decrease in stemness makes IPFP-ASCs a very promising cell source for stem cell-based therapy. In routine cellular therapy approaches, the age of the patient is a crucial parameter that sometimes limits the success of the therapy. For example, human BMSCs isolated from donors of higher age have been shown to be lesser in amount with a reduced lifespan and diminished capacity of proliferation and osteogenic differentiation ${ }^{18)}$. In another study where the osteogenic differentiation potential of ASCs and BMSCs were compared between young (mean \pm standard deviation [SD], $36.4 \pm 11.8$ years) and elderly (mean $\pm \mathrm{SD}, 71.4 \pm 3.6$ years) patients, it was observed that the level of matrix mineralization of ASCs was comparable between young and aged patients, whereas BMSCs from aged patients produced less amount of mineral deposits and had a lower expression level of osteogenic genes as compared to the cells from the young patients ${ }^{19)}$. Although there is no direct comparison of BMSCs to IPFP-ASCs in terms of proliferative and differentiation capacity with aging, the study of Khan et al. ${ }^{15)}$ compared IPFPASCs isolated from patients of different age groups in terms of their proliferative capacity as well as their potential of epitopes and osteogenesis. It was shown in this study that there was no significant difference in proliferation rate at any defined time between cells from different groups. Considering cell surface epitopes, mesenchymal markers including CD13, CD44, CD90, CD105 and CD29 have shown positive staining for all age groups. On the other hand, little or no staining was observed for the following markers: 3G5 for vascular pericytes, LNGFR and STRO-1 for BMSCs and CD34 for hematopoietic stem cells. These suggested a homogenous population and the lack of hematopoietic 
cells during isolation. Negative staining for CD56 (neural cell adhesion molecule marker for neurogenic and myogenic cells) has also revealed the isolation of a pure population without contamination of the cells of the tissues mentioned. As a result, IPFPASCs have shown similar characteristics of MSCs in terms of surface marker expression and these characteristics were maintained with ageing.

In another study, the characteristics and differentiation abilities of IPFP-ASCs and SC-ASCs were examined ${ }^{20}$. It was found that IPFP-ASCs and SC-ASCs had similar cell surface profiles demonstrated by flow cytometry including positive expression of CD73, CD90 and CD105 and negative expression of CD34 and CD45. With respect to differentiation ability, IPFP-ASCs showed superiority in osteogenic and chondrogenic differentiation over SC-ASCs as assessed by SOX-9 (chondrogenic transcription factor) and RUNX-2 (transcription factor expressed in MSCs upon their commitment toward osteogenic differentiation). It was concluded that the anatomical area where cells are isolated affects the characteristics of ASCs, and the level of chondrogenic differentiation potential of IPFP-ASCs is higher due to close contact point of IPFP with synovial membrane and fluid ${ }^{211}$. Therefore, IPFP can be considered as a high quality source for stem cell-based regenerative therapies.

\section{Biological Mechanisms for Regulation of Cell Behavior}

IPFP-ASCs have shown great potential as a cell source in cartilage tissue engineering, where they were shown to fulfill the criteria of chondrogenic potential at a comparable level to MSCs ${ }^{16)}$. Chondrogenic differentiation of ASCs from various sources is usually induced by culturing in differentiation medium containing growth factors such as transforming growth factor- $\beta$ (TGF- $\beta$ ) with its isoforms named as TGF- $\beta 1$, TGF- $\beta 2$ and TGF- $\beta 3^{22)}$. Different procedures have been published so far in the literature in terms of types and effective concentrations of TGF- $\beta$ subtypes. Although there is no report in the literature comparing the effect of three different isoforms of TGF- $\beta$ s on chondrogenic differentiation for IPFP-ASCs, Ye et al. ${ }^{23)}$ showed that culture media consisting of TGF- $\beta 3$ and bone morphogenic protein- 6 (BMP-6) have strongly enhanced the capacity of chondrogenic differentiation of IPFP-ASCs via secretion of collagen type II. Furthermore, the expression of collagen type II and aggrecan located at the extra cellular matrix was confirmed by immunohistochemistry and indicated the hyaline cartilage formation. Hennig et al. ${ }^{24)}$ described the reason behind the synergistic effect of BMP-6 and
TGF- $\beta$ and concluded the contribution of BMP- 6 was to induce the expression of TGF- $\beta$ which is not usually expressed by ASCs.

\section{IPFP-ASCs in Tissue Repair and Regeneration}

The potential of using IPFP-ASCs provides a great promise for the field of orthopedics including cartilage and bone repair. IPFP is a non-negligible source of stem cells for cartilage regeneration due to availability and accessibility of high amount of chondroprogenitor cells within the tissue. Although culturing cells in a three-dimensional (3D) environment is the most common approach for cartilage tissue engineering ${ }^{25}$, this constitutes a significant barrier for the availability of a suitable piece of tissue analogue for transplantation. In order to overcome this problem and to reduce the time and cost related with the in vitro culturing, Ahearne et al. ${ }^{26)}$ developed an approach including direct implantation of TGF- $\beta 1$ delivery system to cartilage defect site for in situ chondrogenesis of IPFP-ASCs encapsulated in fibrin hydrogels. IPFP-ASCs have also been tested in the remediation of articular cartilage defects resulting from osteoarthritis (OA). In the study of Toghraie et al. ${ }^{27}$, IPFP-ASCs were injected in rabbit knees and the lesion site was reduced for IPFP-ASC treated group, while progression of degenerative OA was observed in the non-treated group. In another study, Buckley et al. ${ }^{16)}$ encapsulated IPFPASCs into agarose hydrogel exposed to TGF- $\beta 3$ and applied load to the gel in order to mimic the natural microenvironment of the tissue. As another strategy to produce 3D tissue, IPFP-ASCs combined with TGF- $\beta 3$ and BMP- 6 were $3 \mathrm{D}$ printed within a chitosan scaffold having a pore size of $250 \mu \mathrm{m}^{23)}$. It was observed by gene expressions based on collagen type II and SOX-9 at the end of 4th week of in vitro culture that the gene expressions were significantly upregulated, indicating cartilage formation. IPFPASCs were also used for the generation of a self-assembled cartilage construct on poly ( $\varepsilon$-caprolactone) films ${ }^{28}$. It was shown that tissues formed on these films were rich in sulfated glycosaminoglycan and collagen type II. In addition to the cartilage degeneration, Dragoo et al. ${ }^{29)}$ demonstrated the applicability of IPFP-ASCs encapsulated fibrin masses to repair articular surfaces as well as to provide increased bone regeneration.

The studies mentioned above and some other studies available in the literature that used IPFP-ASCs for regenerative applications in orthopedics are listed in Table 1.

\section{Clinical Applications of ASCs in Orthopedics}

In clinical perspective, patients have benefited from advances 


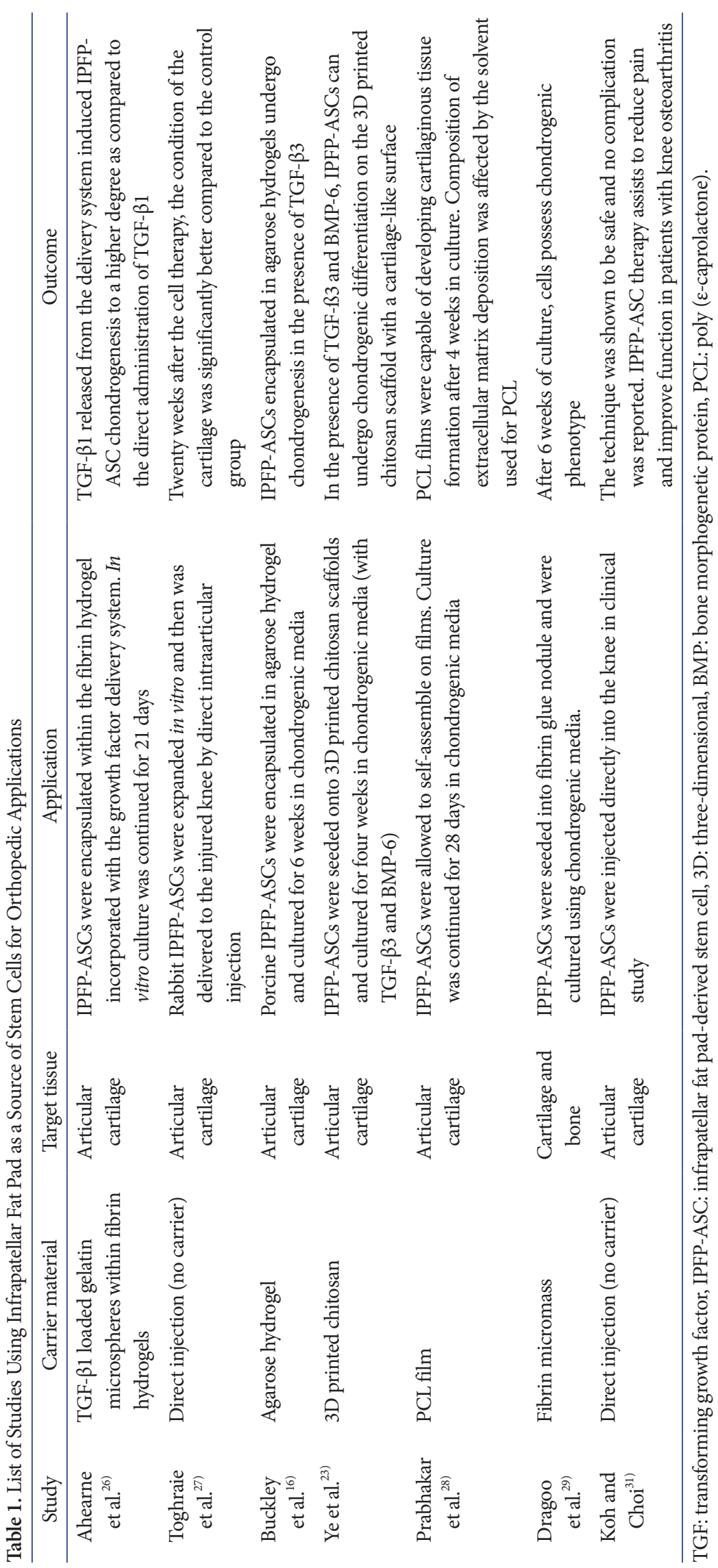


in ASC research in orthopedics. Although IPFP-ASCs have not yet been studied in detail in clinical settings, SC-ASCs have proven the potential in clinical applications in preclinical stud$\mathrm{ies}^{30}$. Therefore, IPFP-ASCs constitute a promising cell source for regenerative therapies of the musculoskeletal tissues in the clinic, regarding their promising preclinical outcomes.

Of the very few examples of clinical use of IPFP-ASCs, the cells were injected percutaneously to determine the potential improvement of articular cartilage with a follow-up period of 1 year $^{31}$. During that period, Lysholm knee scoring, Tegner activity and visual analogue scale (VAS) score were analyzed. After the operation, almost all patients (92\%) showed an improved Lysholm score together with increased VAS score and decreased Tegner activity when compared with pre-operated cases. The results of this study imply encouraging effect of IPFP-ASCs in terms of reducing pain and improving knee function for patients suffering from OA.

Studies involving the use of SC-ASCs in clinical applications include the study of Dufrane et al. ${ }^{32)}$ where human SC-ASCs were used with demineralized bone matrix to form a $3 \mathrm{D}$ "bonelike" osteogenic structure and transplanted to 6 patients having large bone defects. The ideal size of bone defect was modelled and the $3 \mathrm{D}$ graft was put directly into the nonunion part of the bone without any fixation material. In 3 months, the 3D graft promoted bone consolidation and restored the bone anatomy and function without any wound healing, inflammatory reaction or oncological effect.

$\mathrm{Pak}^{33)}$ injected SC-ASCs with hyaluronic acid, platelet rich plasma and $\mathrm{CaCl} 2$ in patients having knee OA. Magnetic resonance imaging (MRI) evidence showed regeneration of the cartilage and bone at 3 months. In another study, Jo et al. ${ }^{34)}$ described the safety and efficacy of intra-articular injection of ASCs for 18 patients having knee OA by six months of follow-up. In order to optimize the dose of injected ASCs, a phase I study was conducted with 3 dose-escalation cohorts which are low-dose $\left(1.0 \times 10^{7}\right.$ cells $)$, mid-dose $\left(5.0 \times 10^{7}\right.$ cells), and high-dose $\left(1.0 \times 10^{8}\right.$ cells) groups, with three patients each. After proving the reliability of ASCs for transplantation, the 9 patients received the high dose in phase II including radiological and histological evaluations. Six months after the injection, the volume of cartilage in medial femoral and tibial condyles increased while the volume of degenerated area of cartilage increased based on MRI. Furthermore, histological analysis (based on safranin O, type I and type II collagen) confirmed thick and hyaline-like cartilage formation integrated with
A

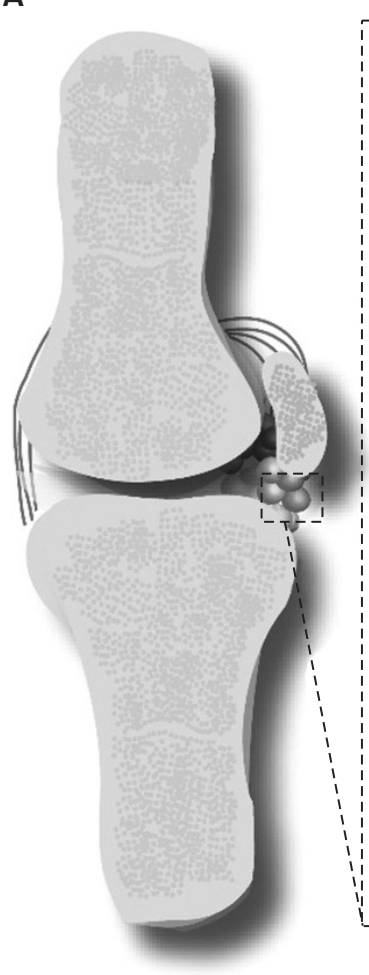

B

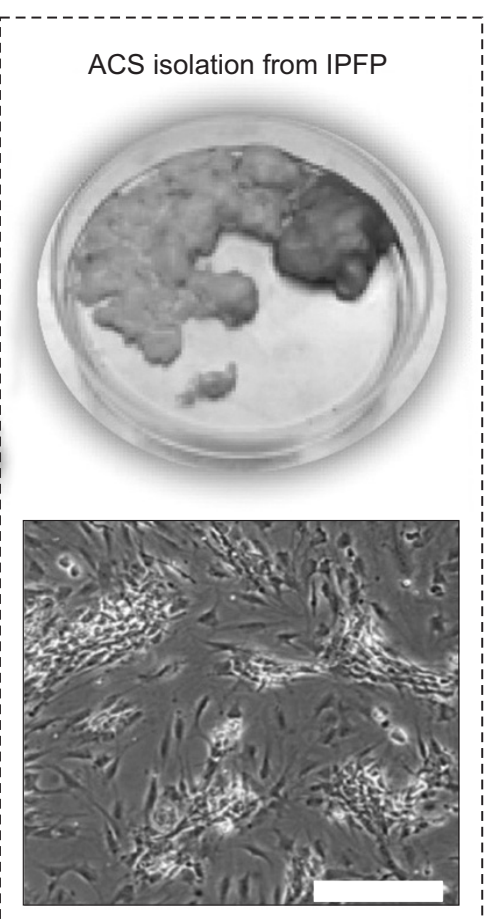

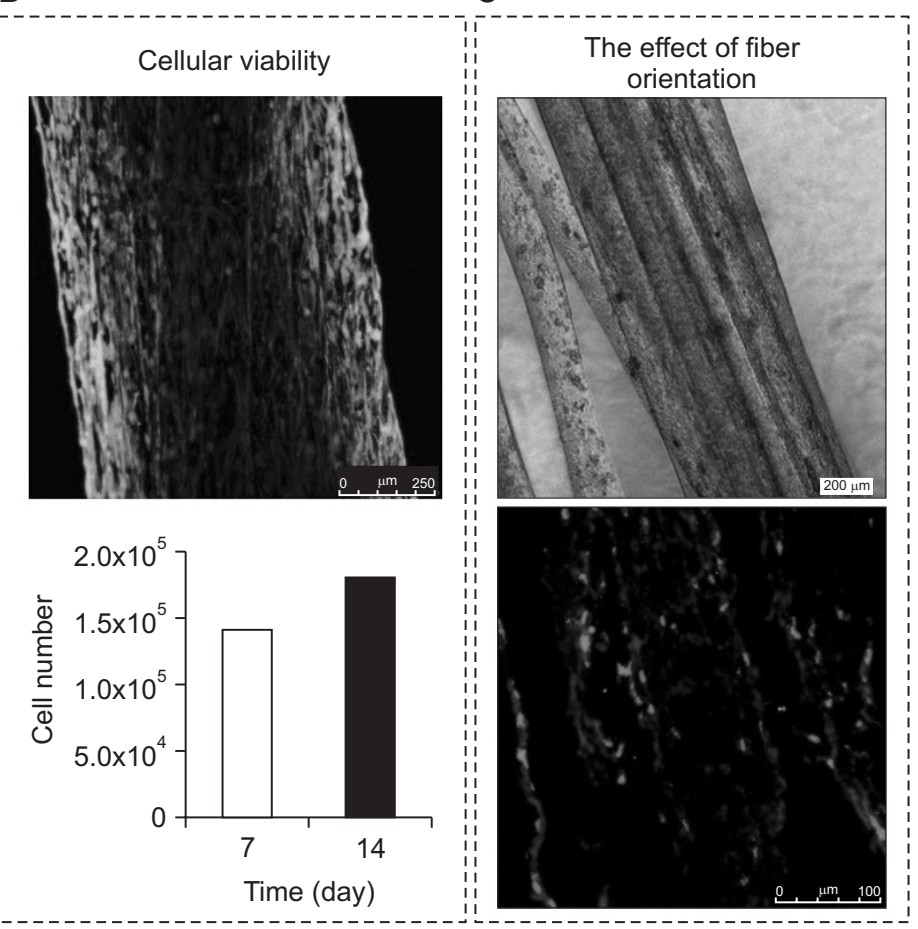

Fig. 1. (A) Infrapatellar fat pad-derived stem cells (IPFP-ASCs) were isolated and encapsulated within wet spun fibrin microfibers. (B) It was observed that the scaffold supported cellular viability for 14 days in the in vitro condition. (C) IPFP-ASCs aligned parallel to the axis of the fibers, which enhanced the expression of muscle-specific marker desmin compared to the non-aligned control (data not shown). 
the subchondral bone. As a result, this study not only optimized the cell dose needed for OA therapy by achieving efficacy and safety but also showed the promising results for cartilage regeneration in clinical perspective.

\section{The Promise of IPFP-ASCs in Tissue Engineering Strategies Targeted towards Alternative Musculoskeletal Tissues}

IPFP-ASCs have shown potential as a stem cell source due to their favorable features as easier accessibility, rapid cell doubling times and anti-apoptotic potential, as mentioned in the above preclinical regenerative strategies mostly towards cartilage. These promising results have fueled the interest to investigate their potential in other target tissues. It has been observed that SC-ASCs have potential as a source of stem cells to develop regenerative strategies for skeletal muscle upon biochemical and mechanical stimulation ${ }^{35}$. Based on this, the potential of IPFP-ASCs for the same target application was investigated. IPFP-ASCs were isolated from IPFP, which was the surgical waste during knee arthroplasty. The myogenic differentiation potential of IPFP-ASCs were investigated for the first time in the literature. Use of IPFPASCs to treat skeletal muscle defects may not become a routine clinical application due to the necessity to damage the joint for cell isolation; however, exploration of the underlying molecular mechanisms would help design better therapeutic approaches towards skeletal muscle regenerative therapies.

Isolated primary IPFP-ASCs were encapsulated within fibrin microfibers produced by wet spinning (Fig. 1A) in order to assess their suitability for use in skeletal muscle tissue engineering. It has been observed that IPFP-ASCs maintain their viability, proliferate and elongate through the axis of the fibers during 14 days of in vitro culture (Fig. 1B). The effect of fiber orientation on the expression of myogenic marker desmin was assessed through immunofluorescence staining performed on cryosections obtained from the cell-laden fibers, counterstained for the cell nuclei with draq5 (Fig. 1C). It was observed that IPFP-ASCs have produced the muscle specific cytoskeletal protein desmin upon biochemical induction.

\section{Conclusion and Future Directions}

Stem cells have shown great potential in regenerative medicine over the last decades. In the field of orthopedics, cell therapy approaches involving the direct injection of stem cells to the target site have been used. However, growing evidence in the literature supports the use of stem cells together with carrier materials of proper biomimetic structure and characteristics in obtaining better regenerative outcomes. Identification of a proper cell source as well as a proper carrier material for these cells are in the heart of successful stem cell-based regenerative strategies. This proper cell source should be accessible, abundant and should maintain proliferative capacity with higher yields of differentiation. Stem cells isolated from adipose tissue (mostly from the abdominal lipoaspirates) are being investigated increasingly as a potent cell source with the mentioned characteristics. ASCs isolated from the IPFP, on the other hand, have shown even better potential than their abdominal counterparts as they possess higher yields in isolation with enhanced proliferation and differentiation capacity. More detailed investigations for the signaling pathways as well as the development of optimized delivery strategies would eventually maximize the efficiency of IPFP-ASCs in their future clinical use in the orthopedic field.

\section{Conflict of Interest}

No potential conflict of interest relevant to this article was reported.

\section{References}

1. Muschler GF, Nakamoto C, Griffith LG. Engineering principles of clinical cell-based tissue engineering. J Bone Joint Surg Am. 2004;86:1541-58.

2. Cancedda R, Dozin B, Giannoni P, Quarto R. Tissue engineering and cell therapy of cartilage and bone. Matrix Biol. 2003;22:81-91.

3. Sharma RR, Pollock K, Hubel A, McKenna D. Mesenchymal stem or stromal cells: a review of clinical applications and manufacturing practices. Transfusion. 2014;54:1418-37.

4. Mizuno H, Tobita M, Uysal AC. Concise review: adiposederived stem cells as a novel tool for future regenerative medicine. Stem Cells. 2012;30:804-10.

5. Gimble J, Guilak F. Adipose-derived adult stem cells: isolation, characterization, and differentiation potential. Cytotherapy. 2003;5:362-9.

6. Siciliano C, Bordin A, Ibrahim M, Chimenti I, Cassiano F, Gatto I, Mangino G, Coccia A, Miglietta S, Bastianelli D, Petrozza V, Calogero A, Frati G, De Falco E. The adipose tissue of origin influences the biological potential of human adipose stromal cells isolated from mediastinal and subcutaneous fat depots. Stem Cell Res. 2016;17:342-51. 
7. Meligy FY, Shigemura K, Behnsawy HM, Fujisawa M, Kawabata $\mathrm{M}$, Shirakawa $\mathrm{T}$. The efficiency of in vitro isolation and myogenic differentiation of MSCs derived from adipose connective tissue, bone marrow, and skeletal muscle tissue. In Vitro Cell Dev Biol Anim. 2012;48:203-15.

8. Varma MJ, Breuls RG, Schouten TE, Jurgens WJ, Bontkes HJ, Schuurhuis GJ, van Ham SM, van Milligen FJ. Phenotypical and functional characterization of freshly isolated adipose tissue-derived stem cells. Stem Cells Dev. 2007;16:91104.

9. Ertas G, Ural E, Ural D, Aksoy A, Kozdag G, Gacar G, Karaoz E. Comparative analysis of apoptotic resistance of mesenchymal stem cells isolated from human bone marrow and adipose tissue. ScientificWorldJournal. 2012;2012: 105698.

10. Vilalta M, Degano IR, Bago J, Gould D, Santos M, GarciaArranz M, Ayats R, Fuster C, Chernajovsky Y, Garcia-Olmo D, Rubio N, Blanco J. Biodistribution, long-term survival, and safety of human adipose tissue-derived mesenchymal stem cells transplanted in nude mice by high sensitivity noninvasive bioluminescence imaging. Stem Cells Dev. 2008;17: 993-1003.

11. Bochev I, Elmadjian G, Kyurkchiev D, Tzvetanov L, Altankova I, Tivchev P, Kyurkchiev S. Mesenchymal stem cells from human bone marrow or adipose tissue differently modulate mitogen-stimulated B-cell immunoglobulin production in vitro. Cell Biol Int. 2008;32:384-93.

12. Dehghanifard A, Shahjahani M, Soleimani M, Saki N. The emerging role of mesenchymal stem cells in tissue engineering. Int J Hematol Oncol Stem Cell Res. 2013;7:46-7.

13. Melief SM, Zwaginga JJ, Fibbe WE, Roelofs H. Adipose tissue-derived multipotent stromal cells have a higher immunomodulatory capacity than their bone marrow-derived counterparts. Stem Cells Transl Med. 2013;2:455-63.

14. Ribeiro A, Laranjeira P, Mendes S, Velada I, Leite C, Andrade P, Santos F, Henriques A, Graos M, Cardoso CM, Martinho A, Pais M, da Silva CL, Cabral J, Trindade H, Paiva A. Mesenchymal stem cells from umbilical cord matrix, adipose tissue and bone marrow exhibit different capability to suppress peripheral blood B, natural killer and T cells. Stem Cell Res Ther. 2013;4:125.

15. Khan WS, Adesida AB, Tew SR, Andrew JG, Hardingham TE. The epitope characterisation and the osteogenic differentiation potential of human fat pad-derived stem cells is maintained with ageing in later life. Injury. 2009;40:150-7.

16. Buckley CT, Vinardell T, Thorpe SD, Haugh MG, Jones E,
McGonagle D, Kelly DJ. Functional properties of cartilaginous tissues engineered from infrapatellar fat pad-derived mesenchymal stem cells. J Biomech. 2010;43:920-6.

17. Doner GP, Noyes FR. Arthroscopic resection of fat pad lesions and infrapatellar contractures. Arthrosc Tech. 2014;3: e413-6.

18. Mendes SC, Tibbe JM, Veenhof M, Bakker K, Both S, Platenburg PP, Oner FC, de Bruijn JD, van Blitterswijk CA. Bone tissue-engineered implants using human bone marrow stromal cells: effect of culture conditions and donor age. Tissue Eng. 2002;8:911-20.

19. Chen HT, Lee MJ, Chen CH, Chuang SC, Chang LF, Ho ML, Hung SH, Fu YC, Wang YH, Wang HI, Wang GJ, Kang L, Chang JK. Proliferation and differentiation potential of human adipose-derived mesenchymal stem cells isolated from elderly patients with osteoporotic fractures. J Cell Mol Med. 2012;16:582-93.

20. Tangchitphisut $P$, Srikaew N, Numhom S, Tangprasittipap A, Woratanarat P, Wongsak S, Kijkunasathian C, Hongeng S, Murray IR, Tawonsawatruk T. Infrapatellar fat pad: an alternative source of adipose-derived mesenchymal stem cells. Arthritis. 2016;2016:4019873.

21. Bobacz K, Sunk IG, Hayer S, Amoyo L, Tohidast-Akrad M, Kollias G, Smolen JS, Schett G. Differentially regulated expression of growth differentiation factor 5 and bone morphogenetic protein 7 in articular cartilage and synovium in murine chronic arthritis: potential importance for cartilage breakdown and synovial hypertrophy. Arthritis Rheum. 2008;58:109-18.

22. Somoza RA, Welter JF, Correa D, Caplan AI. Chondrogenic differentiation of mesenchymal stem cells: challenges and unfulfilled expectations. Tissue Eng Part B Rev. 2014;20:596608.

23. Ye K, Felimban R, Traianedes K, Moulton SE, Wallace GG, Chung J, Quigley A, Choong PF, Myers DE. Chondrogenesis of infrapatellar fat pad derived adipose stem cells in 3D printed chitosan scaffold. PLoS One. 2014;9:e99410.

24. Hennig T, Lorenz H, Thiel A, Goetzke K, Dickhut A, Geiger F, Richter W. Reduced chondrogenic potential of adipose tissue derived stromal cells correlates with an altered TGFbeta receptor and BMP profile and is overcome by BMP-6. J Cell Physiol. 2007;211:682-91.

25. Alves da Silva ML, Crawford A, Mundy JM, Correlo VM, Sol P, Bhattacharya M, Hatton PV, Reis RL, Neves NM. Chitosan/polyester-based scaffolds for cartilage tissue engineering: assessment of extracellular matrix formation. Acta 
Biomater. 2010;6:1149-57.

26. Ahearne M, Buckley CT, Kelly DJ. A growth factor delivery system for chondrogenic induction of infrapatellar fat padderived stem cells in fibrin hydrogels. Biotechnol Appl Biochem. 2011;58:345-52.

27. Toghraie FS, Chenari N, Gholipour MA, Faghih Z, Torabinejad S, Dehghani S, Ghaderi A. Treatment of osteoarthritis with infrapatellar fat pad derived mesenchymal stem cells in Rabbit. Knee. 2011;18:71-5.

28. Prabhakar A, Lynch AP, Ahearne M. Self-assembled infrapatellar fat-pad progenitor cells on a poly- $\varepsilon$-caprolactone film for cartilage regeneration. Artif Organs. 2016;40:376-84.

29. Dragoo JL, Samimi B, Zhu M, Hame SL, Thomas BJ, Lieberman JR, Hedrick MH, Benhaim P. Tissue-engineered cartilage and bone using stem cells from human infrapatellar fat pads. J Bone Joint Surg Br. 2003;85:740-7.

30. Im GI. Clinical use of stem cells in orthopaedics. Eur Cell Mater. 2017;33:183-96.

31. Koh YG, Choi YJ. Infrapatellar fat pad-derived mesenchymal stem cell therapy for knee osteoarthritis. Knee. 2012;19:902-
7.

32. Dufrane D, Docquier PL, Delloye C, Poirel HA, Andre W, Aouassar N. Scaffold-free three-dimensional graft from autologous adipose-derived stem cells for large bone defect reconstruction: clinical proof of concept. Medicine (Baltimore). 2015;94:e2220.

33. Pak J. Regeneration of human bones in hip osteonecrosis and human cartilage in knee osteoarthritis with autologous adipose-tissue-derived stem cells: a case series. J Med Case Rep. 2011;5:296.

34. Jo CH, Lee YG, Shin WH, Kim H, Chai JW, Jeong EC, Kim JE, Shim H, Shin JS, Shin IS, Ra JC, Oh S, Yoon KS. Intra-articular injection of mesenchymal stem cells for the treatment of osteoarthritis of the knee: a proof-of-concept clinical trial. Stem Cells. 2014;32:1254-66.

35. Yilgor Huri P, Cook CA, Hutton DL, Goh BC, Gimble JM, DiGirolamo DJ, Grayson WL. Biophysical cues enhance myogenesis of human adipose derived stem/stromal cells. Biochem Biophys Res Commun. 2013;438:180-5. 